\begin{tabular}{|c|l|}
\hline Title & Multiband Artificial Magnetic Conductors Using Stacked Microstrip Patch Layers \\
\hline Author(s) & Inafune, Koji; Sano, Eiichi \\
\hline Citation & $\begin{array}{l}\text { Communications (A ug. 2005, Beijing, China) } \\
\text { Comme, A ntenna, Propagation and EMC Technologies for Wireless }\end{array}$ \\
\hline Issue Date & 2005 -08 \\
\hline Doc URL & http://hdl.handle.net/2115/13194 \\
\hline Type & proceedings(author version) \\
\hline File Information & 297EMC.pdf \\
\hline
\end{tabular}

Instructions for use 


\title{
Multiband Artificial Magnetic Conductors Using Stacked Microstrip Patch Layers
}

\author{
Koji Inafune and Eiichi Sano \\ Research Center for Integrated Quantum Electronics, Hokkaido University \\ North 13 West 8, Sapporo 060-8628, Japan \\ E-mail: inafune@ rciqe.hokudai.ac.jp, esano@rciqe.hokudai.ac.jp
}

\begin{abstract}
A two-dimensional microstrip patch array with a conducting via connecting the center of each patch to ground has been proposed for an artificial magnetic conductor (AMC). On the other hand, a microstrip patch array without vias has been shown to produce an AMC. This paper analyzes AMC structures with and without vias by using an effective medium model and finite difference time domain (FDTD) full-wave analysis method. The difference between the properties of these AMC structures is investigated in detail. Based on the results, we devised a multiband AMC structure having stacked microstrip patch layers. FDTD calculations and measurements were performed to demonstrate the effectiveness of the multiband AMC.
\end{abstract}

\section{I.Introduction}

Meta-materials such as left-handed systems and artificial magnetic conductors (AMCs), composed of composite materials or circuit components, have been attracting a lot of attention [1]-[4]. A magnetic conductor is in dual relation to an electric conductor, and doesn't exist in nature. An AMC that mimics a_magnetic conductor has been proposed [1]. The surface impedance of an AMC is very high in a frequency region determined by its structure. This property enables us to use AMCs in antenna reflectors [1]. In addition, an active integrated antenna (AIA) structure combining an AMC with varactor diodes achieves a beam steering function [5]. A two-dimensional microstrip patch array with a conducting via connecting the center of each patch to ground has been proposed by Sievenpiper et al. [1]. Moreover, a patch array without vias has been proposed by Zhang et al.[6]. Hereafter, we call the AMC with vias the Sievenpiper structure and the AMC without vias the Zhang structure. The surface impedance of the Sievenpiper structure is very high at the resonance frequency of the $L C$ parallel circuit, where $L$ is the inductance provided by the conducting paths through the vias and the ground plane and $C$ is the capacitance between the neighboring metal patches. The reflection phase on the AMC surface is zero at the resonance frequency. In contrast, there are no explicit inductances in the Zhang structure. It is speculated that these structures have different operation mechanisms. However, no detailed comparison of their electromagnetic behaviors has been made. Previous investigations have focused on the primary resonance frequency between the $\mathrm{TM}_{0}$ and $\mathrm{TE}_{1}$ modes, and little work has been done to realize mutiband AMCs [7]. Kern et al. have proposed fractal geometries to produce mutiband AMCs and presented a design methodology based on a genetic algorithm [7]. However, this method is rather complicated.

The purpose of this paper is to clarify the differences between the electromagnetic behaviors of the Sievenpiper and Zhang structures, and further, to propose multiband AMCs based on a modified Zhang structure having stacked microstrip patch layers. Section II presents an effective medium model and a_finite difference time domain (FDTD) method to analyze the fundamental AMC structures. Calculated results are shown in Section III. Section IV demonstrates theoretical and experimental properties of the proposed multiband AMCs.

\section{Analysis model}

Figure 1 depicts AMC structures and the analysis 


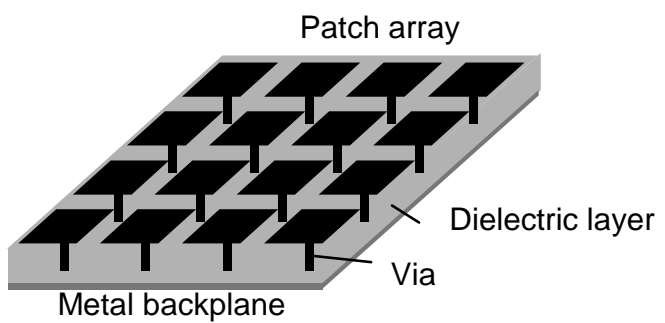

(a)

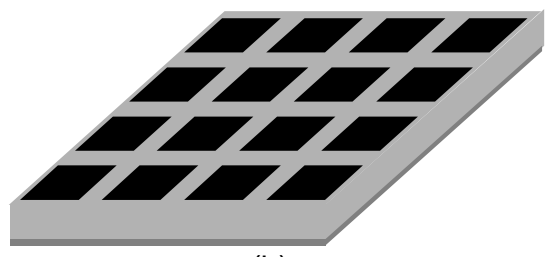

(b)

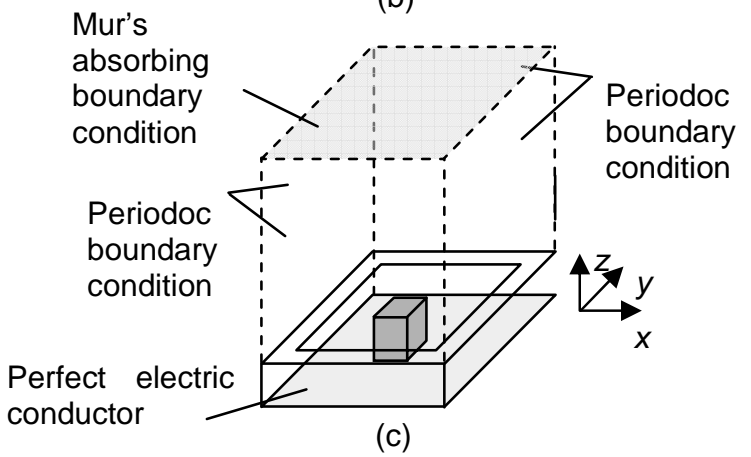

Fig. 1. (a) Sievenpiper structure, (b) Zhang structure and (c) FDTD model.

model for the FDTD method. Dimensions used in the analysis are same as those described in [1]. In the case of the Sievenpiper structure, the period $L$ is $2.4 \mathrm{~mm}$, the spacing between the patches $G$ is 0.15 $\mathrm{mm}$, the via width $S$ is $0.36 \mathrm{~mm}$, the relative permittivity of the substrate $\varepsilon_{D}$ is 2.2 , and the thickness of the substrate $D$ is $1.6 \mathrm{~mm}$. The Zhang structure is the same as the Sivenpiper structure except that it has no vias. The periodic boundary condition is applied to the $y z$ and $z x$ planes. The metal is treated as a perfect conductor with zero thickness. We analyze the reflection characteristics of the AMCs for a normally incident plane wave. The electric field of the incident plane wave is $(0$, $\left.E_{y}, 0\right)$. The reflection phase $\phi$ on the AMC surface is calculated as

$$
\phi=\ln \left(E_{y, \text { ref }} / E_{y, \text { in }}\right)+2 k_{0} l
$$

where $E_{y, r e f}, E_{y, i n}$, and $l$ are reflected wave, incident wave, and the distance between the observed point for $E_{y}$ and the AMC surface, respectively. The

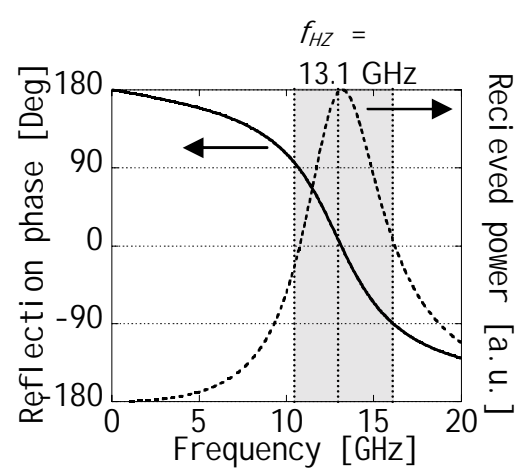

(a)

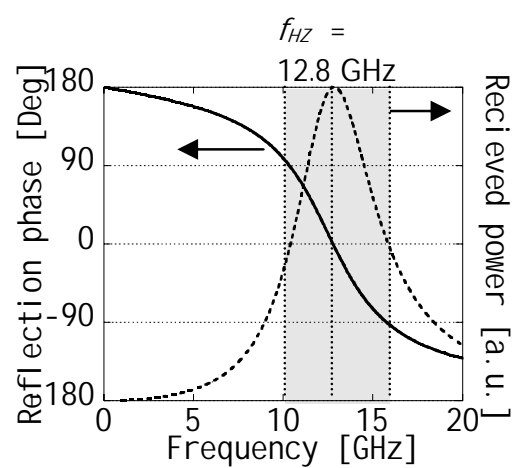

(b)

Fig. 2. Reflection phase and received power of (a) Sievenpiper structure and (b) Zhang structure calculated with FDTD method.

FDTD analysis of the_dispersion characteristics for wave propagation in the $x$-direction was performed by the methodology described in [8].

In the effective medium model, macroscopic effective permittivity and permeability are used for the patch region and substrate region including the via array, because the dimensions of AMC structures are comparable to 1/10th of the operation wavelength. The equations for calculating_effective permittivity and permeability are the same as those described in [9].

\section{Results for Fundamental AMCs}

Figure 2 (a) and (b) show reflection phases and received powers calculated with the FDTD method for the Sievenpiper and Zhang structures. When an incident plane wave enters an AMC surface from the positive $z$ direction, the phase of reflected wave is $\pi$ at very low frequencies, just like an electric perfect conductor. As the frequency increases, the phase decreases, and eventually the wave reflects with zero phase shift at the resonance frequency $f_{H Z}$ of the structure. This is the reason why an AMC is 
also called a "high impedance surface". The $f_{H Z}$ 's of the_Sievenpiper and Zhang structures are 13.1 GHZ and $12.8 \mathrm{GHz}$, respectively. Figure 2 indicates that $f_{H Z}$ is identical to the frequency at which received power peaks. In addition, the received power becomes half of the maximum received power at frequencies where $\phi= \pm \pi / 2$.

The FDTD simulations revealed that each AMC structure first absorbs energy from the incident wave, resonates with it, and then radiates it to free-space, when the plane-wave frequency equals $f_{H Z}$. The time required to perform this process corresponds to a phase of $\pi$, and therefore, the reflection phase becomes zero at $f_{H Z}$. The $f_{H Z}$ 's calculated by the effective medium model for the Sievenpiper and Zhang structures were $14.6 \mathrm{GHz}$ and $12.0 \mathrm{GHz}$, respectively. These results agree with those calculated with the FDTD method within $\sim 10 \%$ accuracy.

Next, we calculated dispersion curves for $x$ directional wave propagation in AMCs by using the effective medium model and FDTD method (Fig.

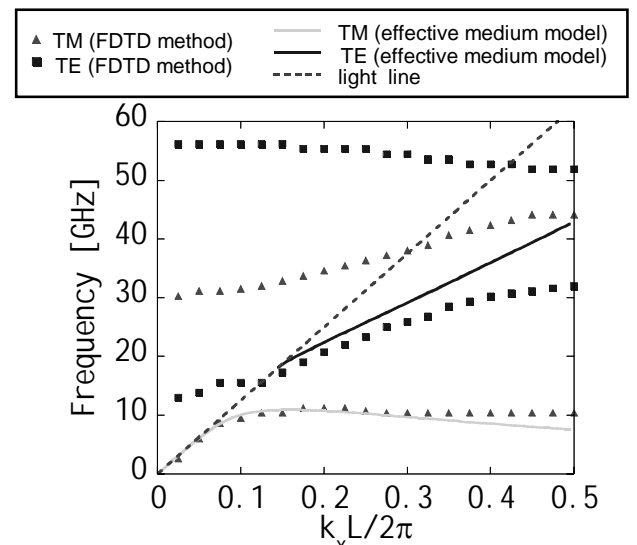

(a)

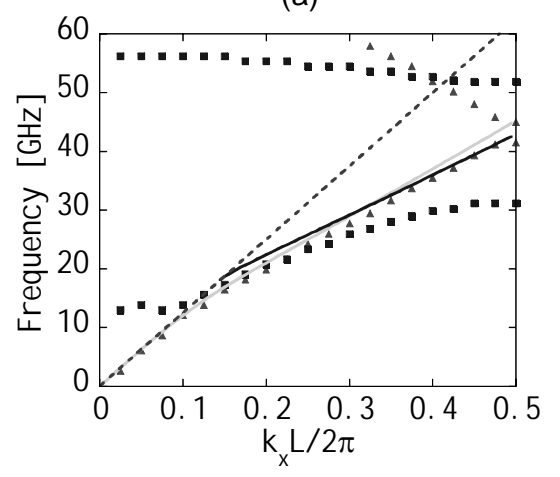

(b)

Fig. 3. Dispersion curves for (a) Sievenpiper structure and

(b) Zhang structure calculated with effective medium model and FDTD method.
3 ). The results obtained with the effective medium model qualitatively agree with those obtained with the FDTD method except in the leaky wave region on the left side of the light line. The curve for the effective medium model deviates from that of the FDTD method as the wavenumber increases, because the macroscopic approximation in the effective medium model deteriorates for large wavenumbers. The main features are as follows: (a) In the Sievenpiper structure, there is a maximum frequency $f_{T M p}$ at a wavenumber $k_{x p}$ for the TM wave. The group velocity $v_{g}$ of the TM wave for $k_{\mathrm{x}}<k_{x p}$ is positive, while $v_{g}$ for $k_{\mathrm{x}}>k_{x p}$ is negative. (b) In the Zhang structure, the dispersion curve for the TM wave increases monotonously. (c) Both structures exhibit similar characteristics for the TE wave. (d) The Sievenpiper structure exhibits a bandgap between $f_{T M p}$ and the TE cutoff frequency $f_{T E c}$. (e) There is no bandgap in the Zhang structure. The measured bandgap of the Sievenpiper structure ranged from $11 \mathrm{GHz}$ to $17 \mathrm{GHz}$ [1], while the bandgap calculated with the effective medium model ranged from $10.9 \mathrm{GHz}$ to $18.1 \mathrm{GHz}$.

\section{Multiband AMC}

We devised a multiband AMC based on the method of enlarging the bandwidth of microstrip patch antennas [10]. Although no bandgap appears in the Zhang structure, its phase characteristics are similar to those for the Sievenpiper structure. Accordingly, we chose the Zhang structure for its fabrication simplicity. Figure 4 shows a schematic view of the

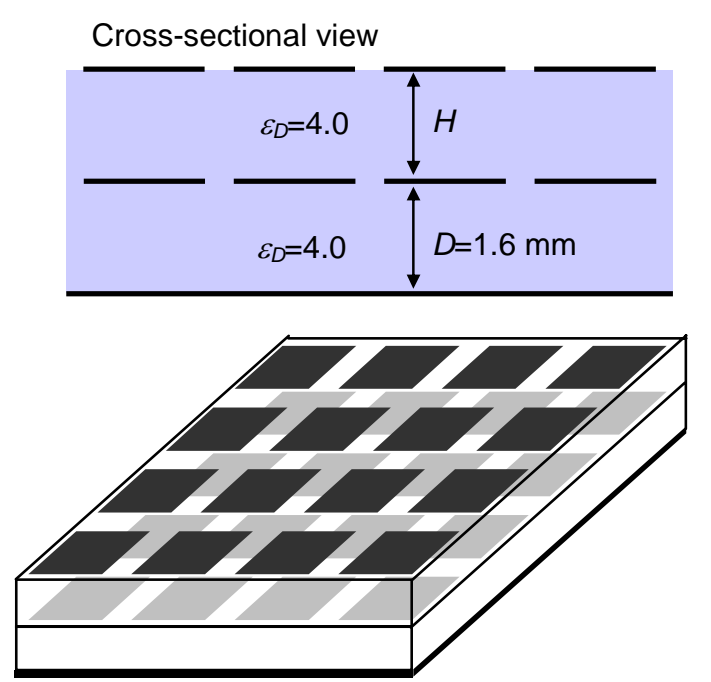

Fig. 4. Structure of multiband AMC using stacked microstrip patch layers. 


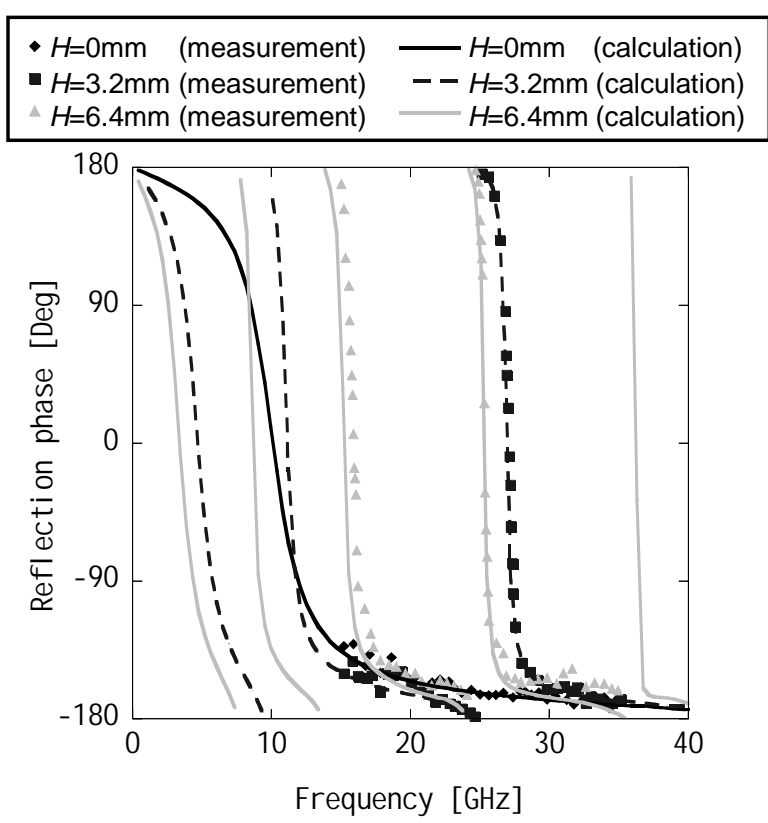

Fig. 5. Measured and calculated phase characteristics for proposed multiband AMCs.

AMCs we fabricated.

The AMCs were fabricated using commercial printed circuit boards. The dimensions of the AMCs were same as those described in Section II. The substrate was FR4. Measurements were performed using a network analyzer and $15-40 \mathrm{GHz}$ antennas. Figure 5 shows the measured reflection phase characteristics for the fabricated AMCs together with those calculated with the FDTD method. Although the measured data are limited to the $15-40 \mathrm{GHz}$ frequency region, the correspondence of the calculations and measurements confirms the effectiveness of the proposed multiband AMCs.

\section{Summary}

AMC structures with and without vias were analyzed with the effective medium model and with the FDTD method. The results showed that reflection phases at the AMC surfaces are zero at their resonance frequencies because the time required for the AMCs to radiate energy absorbed from incident waves corresponds to a_phase of $\pi$. It was found from these calculations that AMC structures with vias exhibit a bandgap, whereas AMCs without vias do not. Based on these results, we devised a multiband AMC structure having stacked microstrip patch layers. Calculations and measurements demonstrated the effectiveness of the proposed multiband AMCs.

\section{Acknowledgments}

This work was partly supported by the Grant-in-Aid for Scientific Research from the Japanese Ministry of Education, Culture, Sports, Science and Technology.

\section{References}

[1] D. Sievenpiper, L. Zhang, R. F. J. Broas, N. G. Alexopolous, and E. Yablonovicth, "High-Impedance Electromagnetic Surfaces with a Forbidden Frequency Band," IEEE Trans. Microwave Theory Tech., vol. 47, no. 11, pp. 2059-2074, Nov 1999.

[2] F. -R. Yang, K. -P. Ma, Y. Qian, and T. Itoh, "A uniplanar compact photonic-bandgap (UC-PBG) structure and its applications for microwave circuit," IEEE Trans. Microwave Theory Tech., vol. 47, no. 8, pp.1509-1514, Aug 1999.

[3] G. V. Eleftheriades, A. K. Iyer, and P. C. Kremer, "Planar Negative Refractive Index Media Using Periodically L-C Loaded Transmission Lines," IEEE Trans. Microwave Theory Tech., vol. 50, no. 12, pp. 2702-2712, Dec 2002.

[4] C. Caloz, A. Sanada, and T. Itoh, "A Novel Composite Right-/Left-Handed Coupled-Line Directional Coupler with Arbitrary Coupling Level and Broad Bandwidth," IEEE Trans. Microwave Theory Tech., vol. 52, no. 3, pp. 980-992, Mar 2004.

[5] D. F. Sievenpiper, J. H. Schaffner, H. J. Song, R. Y. Loo, and G. Tangonan, "Two-Dimensional Beam Steering Using an Electrically Tunable Impedance Surface," IEEE Trans. Antennas Propagat., vol. 51, no. 10, pp. 2713-2722, Oct 2003.

[6] Y. Zhang, J. von Hagen, M. Younis, C. Fischer, and W. Wiesbeck, "Planar Artificial Magnetic Conductors and Patch Antennas," IEEE Trans. Antennas Propagat., vol. 51, no. 10, pp. 2704-2712, Oct 2003.

[7] D. J. Kern, D. H. Werner, A. Monorchio, L. Lanuzza, and M. J. Wilhelm, "The Design of Synthesis of Multiband Artificial Magnetic Conductors Using High Impedance Frequency Selective Surfaces," IEEE Trans. Antennas Propagat., vol. 53, no. 1, pp. 8-17, Jan 2005.

[8] C. T. Chan, Q. L. Yu, and K. M. Ho, "Order-N Spectral Method for Electromagnetic Waves," Appl. Phys. B, vol. 51, no .23, pp. 635-642, June 1995

[9] S. Clavijo, R. E. Diaz, and W. E. McKinzue, III, "Desion Methodo;ogy for Sievenpiper High-Impedance Surfaces: An Artificial Magnetic Conductor for Positive Gain Electrically Small Antennas," IEEE Trans. Antennas Propagat., vol. 51, no. 10, pp. 2678-2690, Oct 2003.

[10] T. Hori, "Broadband/Multiband printed antennas," IEICE Trans. Comm., vol. J87-B, no. 9, pp. 1130-1139, 2004. (in Japanese) 